\title{
ON CERTAIN BOUNDARY-VALUE PROBLEMS WITH STRONG VANISHING ON THE BOUNDARY*
}

\author{
BY \\ J. McCREA AND J. L. SYNGE \\ Dublin Institute for Advanced Studies
}

\begin{abstract}
In Newtonian statics of a continuum we have a symmetric stress tensor with six components and a body-force with three components, and the divergence of the stress tensor equals the body-force vector reversed. If the body-force vector as assigned in some finite domain $I$ with boundary $B$, we have three equations to be satisfied by six stress components. The equations of equilibrium, coupled with conditions on $B$, cannot determine the stress, but they do define a class of stress distributions, provided the body-force and the conditions on $B$ are consistent. The purpose of this paper is to show that, if the body-force satisfies the usual conditions of equilibrium and vanishes strongly on $B$ in the sense that this body-force, and all its derivatives up to order $N$, vanish on $B$, then there exists a stress distribution which also vanishes strongly on $B$, the order of vanishing being greater by one than that of the body-force.

A similar but simpler problem is also discussed. We consider a vector field in $I$ and impose on it the condition that its divergence is equal to a given scalar field which vanishes strongly on $B$ and for which the integral over $I$ vanishes. We show that there exists such a vector field vanishing strongly on $B$ to an order which is one greater than that of the scalar field. This problem may be interpreted physically in terms of a fluid for which the expansion is assigned, or in terms of the thermal expansion of a solid.

As a corollary, we may state that if the body-force (resp. scalar field) is discontinuous on $B$ and zero outside, then we can find a stress distribution (resp. vector field) which vanishes outside and on $B$, although its derivatives do not vanish as we approach $B$ from inside.
\end{abstract}

1. Introduction. The problems considered here arose in an investigation in the general theory of relativity, but as they involve only concepts familiar in Newtonian mechanics, it seems appropriate to present them in that setting. They are somewhat unusual problems since they involve a high degree of indeterminacy (there are more unknowns than equations).

Tensor notation will be used. Greek suffixes take the values 1, 2, 3 and capital Latin suffixes the values 1, 2. Partial derivatives are indicated by commas and covariant derivatives by vertical strokes. We write $x^{\alpha}$ for curvilinear coordinates in Euclidean 3-space, $g_{\mu \nu}$ for the metric tensor and $\Gamma^{\alpha}{ }_{\mu \nu}$ for the Christoffel symbols,

$$
\Gamma^{\alpha}{ }_{\mu \nu}=\frac{1}{2} g^{\alpha \beta}\left(g_{\mu \beta, \nu}+g_{\nu \beta, \mu}-g_{\mu \nu, \beta}\right) .
$$

Let $I$ be a domain in Euclidean 3-space, bounded by a surface $B$, which may be simply or multiply connected. We impose conditions of smoothness on $B$. These conditions are such that there exists, in a layer $L$ of $I$ adjacent to $B$, Gaussian coordinates $x^{\alpha}, x^{3}$ being the normal distance of a point from $B$ and $x^{A}$ being coordinates on $B$, such

*Received March 25, 1966. 
that in $L$ the metric tensor $g_{\mu \nu}$, possesses partial derivatives to some sufficiently high order. To achieve this, it is necessary to take for the coordinates $x^{A}$, not one coordinate system, but several overlapping coordinate systems connected by sufficiently smooth transformations.

These tedious details might be omitted if we were to restrict $B$ to be a sphere, for then it would have the requisite smoothness. However it is desirable to be more general and so we may state as general requirements: (i) differentiability of the metric tensor up to some assigned order, and (ii) the formal properties of Gaussian coordinates, which are

$$
g_{A 3}=g^{A 3}=0, \quad g_{33}=g^{33}=1, \quad \Gamma_{33}^{A}=\Gamma_{A 3}^{3}=\Gamma_{33}^{3}=0 .
$$

Gaussian coordinates based on a surface $B$ (so that the equation of $B$ is $x^{3}=0$ ) are defined only in a layer $L$ which contains no centre of curvature of $B$. These coordinates do not cover the whole interior domain $I$. That is no trouble in our work because that will take place in $L$. But should we wish to pass to the rest of $I$, we would introduce curvilinear coordinates throughout $I$, connecting them smoothly to the Gaussian coordinates in $L$ by means of an overlap. It will be assumed without proof that, if we are given a tensor field in $L$, that field can be extended throughout the whole of $I$.

Evaluation on $B$ will be denoted by a prime set before a main letter: thus ' $f$ means $f$ evaluated on $B$, so that ' $f$ is a function only of the coordinates $x^{A}$.

Definition. We shall say that a function $f$ in $I$ vanishes strongly to order $N$ on $B$ if $f$ and all its partial derivatives, up to and including those of order $N$, vanish on $B$ and we indicate this by writing

$$
\text { 'f } \underset{N}{=} 0 .
$$

Since all the functions appearing in this work may be regarded as being zero outside $B$, (1.3) is equivalent to saying that $f$ is of class $C^{N}$ on $B$.

2. The vector theorem. We shall establish two theorems, a vector theorem and a tensor theorem. Although the details differ, the two theorems are analogous, and, since the vector theorem is the simpler, it will be treated first.

Vector Theorem. Let $K$ be a scalar field in $I$, possessing at least $N$ derivatives and satisfying

$$
\int K d_{3} x=0, \quad ' K \underset{N}{=} 0 .
$$

Then there exists in I a vector field $V^{\alpha}$ satisfying

$$
\left.V^{\alpha}\right|_{\alpha}=K, \quad{ }^{\prime} V^{\alpha}=0 .
$$

To prove this theorem, we start by noting that, by potential theory, there exists a vector field $U_{\alpha}$ (a gradient, but that is not important here) satisfying

$$
\left.U^{\alpha}\right|_{\alpha}=K, \quad{ }^{\alpha} n_{\alpha}=0,
$$

where $n_{\alpha}$ is a normal to $B$. This holds in any curvilinear coordinates. Using Gaussian coordinates, we have

$$
{ }^{\prime} U^{3}=0
$$


while the first of (2.3), written out more explicitly, reads

$$
U^{A}{ }_{{ }_{A}}+U^{3}{ }_{3}+\Gamma^{A}{ }_{M A} U^{M}+\Gamma^{A}{ }_{3 A} U^{3}=K .
$$

Taking this on $B$, and using (2.1) and (2.4), we have

$$
{ }^{\prime} U^{A}{ }_{, A}+{ }^{\prime} U^{3}{ }_{3}+{ }^{\prime} \Gamma^{A}{ }_{M A}{ }^{\prime} U^{M}=0 .
$$

We note that $U^{\alpha}$, although it satisfies the first of (2.2), fails to satisfy the second condition, even for $N=0$, because ${ }^{\prime} U^{A} \neq 0$ in general.

Let us now introduce tentatively a skew tensor field $P^{\alpha \beta}\left(=-P^{\beta \alpha}\right)$ and define in terms of it a vector field

$$
P^{\alpha}=\left.P^{\alpha \beta}\right|_{\beta} .
$$

It is important to note that, since we are working in a flat 3-space, the order in which covariant derivations are applied is immaterial

Hence

$$
\left.X \cdots\right|_{\alpha \beta}=\left.X \cdots\right|_{\beta \alpha} .
$$

$$
\left.P^{\alpha}\right|_{\alpha}=\left.P^{\alpha \beta}\right|_{\beta \alpha}=-\left.P^{\beta \alpha}\right|_{\alpha \beta}=-\left.P^{\beta}\right|_{\beta}
$$

and so

$$
\left.P^{\alpha}\right|_{\alpha}=0 .
$$

Leaving $P^{\alpha \beta}$ for the moment free (except for skew-symmetry), let us set

$$
V^{\alpha}=U^{\alpha}-P^{\alpha},
$$

where $U^{\alpha}$ is as above. It is clear that $V^{\alpha}$ satisfies the first of (2.2), and our task is to choose $P^{\alpha \beta}$ so that the second of (2.2) is also satisfied. Since our aim is to establish the Vector Theorem by finding some suitable $P^{\alpha \beta}$, not the most general, we shall simplify the calculations by choosing once for all

$$
P^{A B}=0,
$$

so that we have only the two functions $P^{A 3}$ to choose.

We must now express $P^{\alpha}$ in terms of those quantities. By (2.7)

$$
P^{\alpha}=P^{\alpha \beta}{ }_{\beta}+\Gamma^{\beta}{ }_{\mu \beta} P^{\alpha \mu},
$$

and, with (1.2) and (2.12), this gives

$$
P^{A}=P^{A 3}{ }_{3}+\Gamma^{B}{ }_{3 B} P^{A 3}, \quad P^{3}=P^{3 B}{ }_{B}+\Gamma^{B}{ }_{M B} P^{3 M} .
$$

The plan will now be to prove the Vector Theorem for $N=0$, and then to proceed by induction, assuming the theorem true for $N$ and proving that it is then true for $(N+1)$.

3. The vector theorem for $N=0$. Extracting the essentials from (2.1) and (2.2), the Vector Theorem for $N=0$ states that if

$$
' K=0,
$$

then $V^{\alpha}$ exists satisfying

$$
\left.V^{\alpha}\right|_{\alpha}=K, \quad ' V^{\alpha}=0
$$


In terms of the machinery set up in the preceding section, this means that we are seeking $P^{A 3}$ such that (cf. (2.11))

$$
{ }^{\prime} P^{\alpha}={ }^{\prime} U^{\alpha},\left.\quad ' P^{\alpha}\right|_{\beta}=\left.{ }^{\prime} U^{\alpha}\right|_{\beta} .
$$

We shall first of all satisfy the first of (3.3), which is equivalent to (cf. (2.4))

$$
{ }^{A}={ }^{A} U^{A}, \quad ' P^{3}=0 .
$$

Let us choose, as values on $B$,

$$
{ }^{\prime} P^{A 3}=0, \quad{ }^{A 3}{ }_{3}={ }^{\prime} U^{A} .
$$

Now (2.14) hold on $B$, and so we may rewrite these equations with ' $P$ for $P$. Inserting the values (3.5), we see at once that the required conditions (3.4) are satisfied. In other words, by (3.5)

$$
{ }^{\prime} P^{\alpha}=\left.{ }^{\prime} P^{\alpha \beta}\right|_{\beta}={ }^{\prime} U^{\alpha},
$$

which immediately implies that

$$
{ }^{\prime} P^{\alpha}{ }_{, A}={ }^{\prime} U^{\alpha}{ }_{, A} .
$$

Using (3.6) and (3.7), the second of (3.3) reduces to

$$
{ }^{\prime} P^{\alpha}{ }_{3}={ }^{\prime} U^{\alpha}{ }_{3} \text {. }
$$

For $\alpha=3,(3.8)$ is automatically satisfied, for if we differentiate the second of (2.14) with respect to $x^{3}$, substitute (3.5) and use the first of (2.3) along with (3.1) we obtain

$$
{ }^{3} P^{3}{ }_{3}={ }^{\prime} U^{3}{ }_{3} \text {. }
$$

In order to satisfy (3.8) for $\alpha=A$, let us further restrict $P^{43}$, by choosing, in addition to $(3.5)$,

$$
{ }^{\prime A 3}{ }_{, 33}={ }^{\prime} U^{A}{ }_{, 3}-{ }^{B} \Gamma_{3 B}{ }^{\prime} U^{A} .
$$

Then, differentiating the first of (2.14) with respect to $x^{3}$ and substituting (3.10), we obtain

$$
{ }^{\prime} P^{A}{ }_{3}={ }^{\prime} U^{A}{ }_{3} .
$$

We have now satisfied (3.3) and so we have proved the Vector Theorem for $N=0$ by putting

$$
\begin{gathered}
V^{\alpha}=U^{\alpha}-P^{\alpha}, \quad P^{\alpha}=\left.P^{\alpha \beta}\right|_{\beta}, \quad P^{A B}=0, \quad{ }^{A 3}=0, \\
{ }^{\prime} P^{A 3}{ }_{3}={ }^{\prime} U^{A}, \quad{ }^{A} P^{A 3}{ }_{, 33}={ }^{\prime} U^{A}{ }_{, 3}-{ }^{\prime} \Gamma^{B}{ }_{3 B}{ }^{\prime} U^{A} .
\end{gathered}
$$

We might write

$$
P^{A 3}=x^{3 \prime} U^{A}+\frac{1}{2}\left(x^{3}\right)^{2}\left({ }^{\prime} U^{A}{ }_{3}-{ }^{\prime} \Gamma^{B}{ }_{3 B}{ }^{\prime} U^{A}\right) \text { in } L,
$$

thus expressing these quantities as functions of all three coordinates.

To complete the construction, these functions are to be extended smoothly into the rest of $I$, and we assume that this presents no difficulty.

Note that we have chosen values for

$$
' P^{\alpha \beta},\left.\quad P^{\alpha \beta}\right|_{\epsilon_{1}},\left.\quad ' P^{\alpha \beta}\right|_{\epsilon_{1} \epsilon_{3}} .
$$


4. Induction for the vector theorem $(N \rightarrow N+1)$. We have proved the vector theorem for $N=0$ and, in doing so, have established the existence of ' $P^{\alpha \beta},{ }^{\prime} P^{\alpha \beta}{ }_{\text {, }}$ and $\left.{ }^{\prime} P^{\alpha \beta}\right|_{\epsilon_{1} \epsilon_{2}}$ such that, with $P^{\alpha}=\left.P^{\alpha \beta}\right|_{\beta}$ as always, ${ }^{\prime} P^{\alpha}={ }_{1}{ }^{\prime} U^{\alpha}$ and hence ${ }^{\prime} V^{\alpha}={ }_{1} 0$, as required in (3.2).

Let us now assume the Vector Theorem for some value of $N$ and that we have established the existence of

$$
' P^{\alpha \beta},\left.{ }^{\alpha \beta}\right|_{\epsilon_{1}}, \cdots,\left.{ }^{\alpha \beta}\right|_{\epsilon_{1} \cdots \epsilon_{N+3}} .
$$

such that, with $P^{\alpha}=\left.P^{\alpha \beta}\right|_{\beta}$, we have

$$
' P^{\alpha}={ }^{\prime} U^{\alpha},\left.\quad{ }^{\alpha}\right|_{\epsilon_{2}}=\left.{ }^{\prime} U^{\alpha}\right|_{\epsilon_{1}}, \cdots,\left.{ }^{\prime} P^{\alpha}\right|_{\epsilon_{1} \cdots \epsilon_{N+1}}=\left.{ }^{\prime} U^{\alpha}\right|_{\epsilon_{1}} \cdots \epsilon_{N+1}
$$

so that, with $V^{\alpha}=U^{\alpha}-P^{\alpha}$ as always, we have

$$
{ }^{\prime} V^{\alpha}=0,\left.\quad{ }^{\prime} V^{\alpha}\right|_{\epsilon_{1}}=0,\left.\cdots{ }^{\prime} V^{\alpha}\right|_{\epsilon_{1} \cdots \epsilon_{N+1}}=0,
$$

these being the conditions demanded in (2.2) at stage $N$, and already satisfied for $N=0$.

Our task is to choose

$$
\left.' P^{\alpha \beta}\right|_{\epsilon_{1} \cdots \epsilon_{N+3}}
$$

(other than those of these quantities already assigned) so as to make

$$
\left.{ }^{\prime} V^{\alpha}\right|_{\epsilon_{1} \cdots \epsilon_{N+2}}=0 \text {, }
$$

or, equivalently,

$$
\left.{ }^{\prime} P^{\alpha}\right|_{\epsilon_{1} \cdots \epsilon_{N+3}}=\left.{ }^{\prime} U^{\alpha}\right|_{\epsilon_{1} \cdots \epsilon_{N+2}},
$$

being given that

$$
' K \underset{N+1}{=} 0 .
$$

Noting that (4.2) are equivalent to the same equations with the stroke replaced by a comma, it is easy to see (cf. (3.6) and (3.7)) that (4.6) are automatically satisfied unless all the $\epsilon$ 's are set equal to 3 , and that in fact, in view of (4.2), all we have to satisfy is

$$
{ }^{\prime} P^{\alpha}{ }_{3(N+2) 3}={ }^{\alpha} U^{\alpha}{ }_{3(N+2) 3},
$$

this notation indicating the presence of $(N+2) 3$ 's.

It is convenient at this point to prove the following lemma:

Lemma. (4.8) is satisfied automatically for $\alpha=3$ by virtue of (4.2).

The proof of this lemma is important, since it brings in an idea which will be useful when we come to the Tensor Theorem. It has already been shown in Sec. 3 for the case $N=0$. We recall the identity $(2.10)$,

$$
\left.P^{\alpha}\right|_{\alpha}=0,
$$

and the differential equation (2.3),

$$
\left.U^{\alpha}\right|_{\alpha}=K \text {. }
$$

Let us take covariant derivatives and proceed to the boundary $B$, remembering that $K$ vanishes strongly there to order $N+1$ (cf. (4.7)). Let us also recall that order is immaterial in covariant differentiation. Thus we get

$$
\left.{ }^{\prime} P^{\alpha}\right|_{\epsilon_{2} \cdots \epsilon_{N+2} \alpha}=\left.{ }^{\prime} U^{\alpha}\right|_{\epsilon_{1} \cdots \epsilon_{N+1} \alpha}=0 .
$$


Now, expanding the last covariant derivative, we have

$$
\begin{aligned}
& \left.{ }^{\prime} P^{\alpha}\right|_{\epsilon_{1} \cdots \epsilon_{N+1} \alpha}=\left.{ }^{\prime} P^{\alpha}\right|_{\epsilon_{1} \cdots \epsilon_{N+1}, \alpha}+\text { other terms, } \\
& \left.{ }^{\prime} U^{\alpha}\right|_{\epsilon_{1} \cdots \epsilon_{N+1} \alpha}=\left.{ }^{\prime} U^{\alpha}\right|_{\epsilon_{1} \cdots \epsilon_{N+1}, \alpha}+\text { other terms, }
\end{aligned}
$$

where the "other terms" involve only derivatives of order $N+1$ and so, by (4.2), are the same in each of the two cases. Hence (4.11) gives

$$
\left.' P^{\alpha}\right|_{\epsilon_{1} \ldots \epsilon_{N+1}, \alpha}=\left.{ }^{\prime} U^{\alpha}\right|_{\epsilon_{1} \cdots \epsilon_{N+1}, \alpha} .
$$

But since (4.2) hold all over $B$, we can differentiate them with respect to $x^{1}$ and $x^{2}$, and so (4.13) reduces to

$$
\left.' P^{3}\right|_{\epsilon_{1} \cdots \epsilon_{N+1}, 3}=\left.{ }^{\prime} U^{3}\right|_{\epsilon_{1} \cdots \epsilon_{N+1}, 3} .
$$

If we now expand out the covariant derivatives and make use of (4.2) (with commas for strokes), we see that (4.14) is automatically satisfied unless all the $\epsilon$ 's are 3, and in fact we are left with

$$
P^{3}{ }_{, 3(N+2) 3}={ }^{3}{ }^{3}{ }_{3(N+2) 3} .
$$

The lemma is proved.

Thus to make the induction from $N$ to $(\mathrm{N}+1)$, all we need is to satisfy

$$
P^{A}{ }_{.3(N+2) 3}={ }^{A} U^{A}{ }_{3(N+2) 3},
$$

by proper choice of

$$
P^{A 3}{ }_{3(N+3) 3} \text {. }
$$

In fact we have to satisfy

$$
\left.' P^{A \beta}\right|_{\beta, 3(N+2) 3}={ }^{A}{ }_{, 3(N+2) 3} .
$$

Expanding the left hand side, we have

$$
{ }^{\prime} P^{A 3}{ }_{3(N+3) 3}+\text { other terms }={ }^{\prime} U^{A}{ }_{3(N+2) 3},
$$

wherein the "other terms" have already been assigned by virtue of (4.1). Thus all we have to do is to read off the value of (4.17) from (4.19), and from (4.17) and earlier assignments we get the quantities (4.4) so as to satisfy (4.5).

Thus the proof of the induction $(N \rightarrow(N+1))$ is completed, and so the Vector Theorem is proved, since it was already proved for $N=0$.

5. The tensor theorem. Although the Tensor Theorem discussed below is, in a sense, a generalization of the Vector Theorem, proved above, it requires a separate proof. We have to start again on the basis laid down in Sec. 1.

Tensor Theorem. Let $K^{\alpha}$ be a vector field in $I$, possessing at least $N$ derivatives and satisfying

$$
\int \xi_{\alpha} K^{\alpha} d_{3} x=0, \quad ' K^{\alpha}=0,
$$

where $\xi_{\alpha}$ is an arbitrary Killing vector $\left(\left.\xi_{\alpha}\right|_{\beta}+\left.\xi_{\beta}\right|_{\alpha}=0\right)$. Then there exists in I a symmetric tensor field $T^{\alpha \beta}$ satisfying

$$
\left.T^{\alpha \beta}\right|_{\beta}=K^{\alpha}, \quad ' T^{\alpha \beta} \underset{N+1}{=} 0 .
$$


The first of (5.1) will be recognized as the usual set of six conditions for statical equilibrium under body-force $-K^{\alpha}$. The theorem asserts the existence of a stress-system for a body in equilibrium under that body-force, and the possibility of finding such a stress-system so that it vanishes strongly on the boundary to an order which is greater by one than that of the body-force.

Problems of statical equilibrium are usually considered for elastic bodies, for which there is a constitutive equation connecting stress and strain, and the problem is determinate. Here we have no constitutive equation, and the determination of the stress is highly indeterminate (3 equations for 6 unknowns).

However, to make the first step towards proving the theorem, we assume a constitutive equation as in elasticity, and accept from that theory the existence of a stress $S^{\alpha \beta}$ consistent with zero traction across the boundary $B$. Thus, just as we had in (2.3) a vector field $U^{\alpha}$, we now have a symmetric tensor field $S^{\alpha \beta}$ satisfying

$$
\left.S^{\alpha \beta}\right|_{\beta}=K^{\alpha}, \quad{ }^{\alpha \beta} n_{\beta}=0 .
$$

Thus in Gaussian coordinates we have

$$
{ }^{\prime} S^{\alpha 3}=0,
$$

and the first of (5.3) gives

$$
S^{\alpha B}{ }_{, B}+S^{\alpha 3}{ }_{3}+\Gamma^{\alpha}{ }_{\mu \beta} S^{\mu \beta}+\Gamma^{\beta}{ }_{\mu \beta} S^{\alpha \mu}=K^{\alpha} .
$$

Proceeding to $B$ and using (5.4), we get

$$
\begin{aligned}
{ }^{\prime} S^{A B}{ }_{B B}+{ }^{\prime} S^{A 3}{ }_{3}+{ }^{\prime} \Gamma^{A}{ }_{M B}{ }^{\prime} S^{M B}+{ }^{\prime B} \Gamma^{B}{ }_{M B}{ }^{\prime} S^{A M} & =0, \\
{ }^{\prime} S^{33}{ }_{33}+{ }^{\prime} \Gamma^{3}{ }_{M B}{ }^{\prime} S^{M B} & =0 .
\end{aligned}
$$

In dealing with the Vector Theorem, we introduced at (2.7) a skew tensor field $P^{\alpha \beta}$. Following Dorn and Schild (1956), we now introduce a tensor field $Q^{\alpha \beta \gamma \delta}$ with the following symmetry:

$$
Q^{\alpha \beta \gamma \delta}=Q^{\gamma \delta \alpha \beta}=-Q^{\beta \alpha \gamma \delta} .
$$

(This is the same symmetry as that of the Riemann tensor, except that the cyclic condition is not assumed.) Further, where previously we introduced a vector $P^{\alpha}$, now we introduce a tensor

$$
Q^{\alpha \gamma}=\left.Q^{\alpha \beta \gamma \delta}\right|_{\beta \delta} .
$$

It is easy to see that

$$
Q^{\alpha \gamma}=Q^{\gamma \alpha}
$$

and

$$
\left.Q^{\alpha \gamma}\right|_{\gamma}=0
$$

identically.

If we now set

$$
T^{\alpha \beta}=S^{\alpha \beta}-Q^{\alpha \beta},
$$

it is clear that this satisfies the first of (5.2), and our task is to choose $Q^{\alpha \beta \gamma \delta}$ so as to satisfy the second of (5.2). 
In the vector case, we simplified the work by (2.12). Likewise we now take, once for all,

$$
Q^{A B C D}=0, \quad Q^{3 B C D}=0 .
$$

Defining

$$
W^{\alpha \gamma}=Q^{\alpha 3 \gamma 3}=W^{\gamma \alpha},
$$

we have

$$
W^{A C}=Q^{A 3 C 3}, \quad W^{\alpha 3}=0,
$$

and we now have only three disposable functions, $W^{A C}$.

Just as in (2.14) we expressed $P^{\alpha}$ in terms of $P^{A 3}$, now we must express $Q^{\alpha \gamma}$ in terms of $W^{A C}$. This is a more formidable proceeding, on account of the second-order covariant derivative, but the work is straightforward. However the details of the result are not needed, and it suffices to write it as follows:

$$
\begin{aligned}
& Q^{A C}=W^{A C}{ }_{, 33}+\left(\begin{array}{cc}
A & C \\
M & N
\end{array}\right) W_{{ }_{3}}^{M N}+E^{A C}, \\
& Q^{\alpha 3}=\left(\begin{array}{c}
\alpha \\
M N
\end{array}\right) W_{{ }_{3}}^{M N}+\left(\begin{array}{c}
\alpha P \\
M N
\end{array}\right) W_{{ }_{3} P}^{M N}+F^{\alpha},
\end{aligned}
$$

the parentheses depending only on the Christoffel symbols $(\Gamma)$ and $E^{A C}$ and $F^{\alpha}$ being linear homogeneous expressions in $W^{M N}, W^{M N}{ }_{, P}, W^{M N}{ }_{P Q}$ with coefficients depending only on the $\Gamma$ 's.

As in the case of the Vector Theorem, we shall first prove the Tensor Theorem for $N=0$, and then proceed by induction.

6. The tensor theorem for $N=0$. Extracting the essentials from (5.1) and (5.2), the Tensor Theorem for $N=0$ asserts that if

$$
' K^{\alpha}=0,
$$

then $T^{\alpha \beta}$ exists satisfying

$$
\left.T^{\alpha \beta}\right|_{\beta}=K^{\alpha}, \quad ' T^{\alpha \beta}=0 .
$$

To construct such a solution, we seek $W^{A C}$ such that (cf. (5.11))

$$
{ }^{\prime} Q^{\alpha \beta}={ }^{\prime} S^{\alpha \beta},\left.\quad{ }^{\alpha \beta}\right|_{\epsilon}=\left.{ }^{\prime} S^{\alpha \beta}\right|_{\epsilon} .
$$

We shall first of all satisfy the first of (6.3) which is equivalent to (cf. (5.4))

$$
{ }^{\prime} Q^{A C}={ }^{\prime} S^{A C}, \quad{ }^{\alpha 3}=0 .
$$

Let us choose

$$
{ }^{\prime} W^{A C}=0, \quad{ }^{A} W^{A C}{ }_{3}=0, \quad{ }^{A} W^{A C}{ }_{33}={ }^{\prime} S^{A C} .
$$

Then taking (5.15) on $B$, the right hand sides vanish except for one term and we get for ' $Q^{\alpha \beta}$ precisely the values required by (6.4).

Using the first of (6.3), which we have satisfied, and the fact that the equality of two functions over the surface implies the equality of their derivatives in the surface, 
we find that the second of $(6.3)$ reduces to

$$
{ }^{\prime} Q^{\alpha \gamma}{ }_{, 3}={ }^{\prime} S^{\alpha \gamma}{ }_{3} \text {. }
$$

For $\gamma=3,(6.6)$ is automatically satisfied, for by (5.3), (5.10) and (6.1)

$$
\left.{ }^{\prime} Q^{\alpha \gamma}\right|_{\gamma}=\left.{ }^{\prime} S^{\alpha \gamma}\right|_{\gamma},
$$

and so by the first of $(6.3)$

$$
{ }^{\prime} Q^{\alpha 3}{ }_{3}={ }^{\prime} S^{\alpha 3}{ }_{3} .
$$

In order to satisfy (6.6) for $(\alpha \gamma)=(A C)$, we further restrict $W^{A C}$ by choosing (cf. (5.15))

$$
{ }^{\prime} W^{A C}{ }_{333}={ }^{4} S^{4 C}{ }_{3}-'\left(\begin{array}{cc}
A & C \\
M & N
\end{array}\right) S^{M N} .
$$

Using (5.15) and (6.5), it is easy to verify that

$$
{ }^{\prime} Q^{A C}{ }_{, 3}={ }^{\prime} S^{A C}{ }_{3} \text {. }
$$

Thus the Tensor Theorem is proved for $N=0$.

Note that we have chosen values of

$$
' Q^{\alpha \beta \gamma \delta},\left.\quad{ }^{\alpha \beta \gamma \delta}\right|_{\epsilon_{1}},\left.\quad{ }^{\alpha \beta \gamma \delta}\right|_{\epsilon_{1} \epsilon_{2}},\left.\quad{ }^{\alpha \beta \gamma \delta}\right|_{\epsilon_{1} \epsilon_{2} \epsilon_{3}},
$$

for these values follow from the values in (6.5) and (6.9), with (5.12).

7. Induction for the tensor theorem $(N \rightarrow(N+1))$. To prove the induction $N \rightarrow(N+1)$ for the Tensor Theorem, we shall follow the same general lines as those followed in Sec. 4 for the Vector Theorem. Thus we assume that we already have values for

$$
' Q^{\alpha \beta \gamma \delta},\left.\quad{ }^{\alpha \beta \gamma \delta}\right|_{\epsilon_{1}},\left.\quad \cdots \quad{ }^{\alpha \beta \gamma \delta}\right|_{\epsilon_{1} \cdots \epsilon_{N+3}}
$$

(cf. (6.11) for $N=0$ ), satisfying certain requirements, as follows. When, by the definition

$$
Q^{\alpha \gamma}=\left.Q^{\alpha \beta \gamma \delta}\right|_{\beta \delta},
$$

and the covariant derivatives of this equation, we calculate values on $B$, we have

$$
' Q^{\alpha \gamma}={ }^{\prime} S^{\alpha \gamma},\left.\quad{ }^{\alpha \gamma}\right|_{\epsilon_{2}}=\left.{ }^{\prime} S^{\alpha \gamma}\right|_{\epsilon_{1}},\left.\quad \cdots \quad{ }^{\prime} Q^{\alpha \gamma}\right|_{\epsilon_{1} \cdots \epsilon_{N+1}}=\left.{ }^{\prime} S^{\alpha \gamma}\right|_{\epsilon_{1} \cdots \epsilon_{N+1}}
$$

so that, with $T^{\alpha \gamma}=S^{\alpha \gamma}-Q^{\alpha \gamma}$ as always, we have

$$
' T^{\alpha \gamma}=0,\left.\quad{ }^{\prime} T^{\alpha \gamma}\right|_{\epsilon_{1}}=0,\left.\quad \cdots \quad ' T^{\alpha \gamma}\right|_{\epsilon_{1} \cdots \epsilon_{N+1}}=0,
$$

these being the conditions demanded in (5.2) and already satisfied for $N=0$.

Our task is to choose

$$
\left.' Q^{\alpha \beta \gamma \delta}\right|_{\epsilon_{1} \cdots \epsilon_{N+}}
$$

(other than those of these quantities already assigned) so as to make

$$
\left.{ }^{\prime} T^{\alpha \gamma}\right|_{\epsilon_{1} \cdots \epsilon_{N+2}}=0
$$

or, equivalently,

$$
\left.' Q^{\alpha \gamma}\right|_{\epsilon_{1} \cdots \epsilon_{N+2}}=\left.{ }^{\prime} S^{\alpha \gamma}\right|_{\epsilon_{1} \cdots \epsilon_{N+2}},
$$


being given that

$$
' K^{\alpha} \underset{N+1}{=} 0 .
$$

We now recall the identity (5.10),

$$
\left.Q^{\alpha \gamma}\right|_{\gamma}=0
$$

and the equation (5.3),

$$
\left.S^{\alpha \gamma}\right|_{\gamma}=K^{\alpha}
$$

and from these we derive, using (7.8),

$$
\left.{ }^{\prime} Q^{\alpha \gamma}\right|_{\epsilon_{1} \cdots \epsilon_{N+1} \gamma}=\left.{ }^{\prime} S^{\alpha \gamma}\right|_{\epsilon_{1} \cdots \epsilon_{N+1} \gamma}=0 .
$$

In view of (7.3), these equations yield

$$
{ }^{\prime} Q^{\alpha 3}{ }_{, 3(N+2) 3}={ }^{\prime} S^{\alpha 3}{ }_{, 3(N+2) 3},
$$

the argument being much as in Sec. 4. Comparing these with the required equations (7.7), we see that the latter are automatically satisfied except perhaps for

$$
{ }^{\prime} Q^{A C}{ }_{3(N+2) 3}={ }^{A} S^{A C}{ }_{3(N+2) 3},
$$

or equivalently,

$$
\left.{ }^{\prime} Q^{A \beta C \delta}\right|_{\beta \delta, 3(N+2) 3}={ }^{A} S^{A C}{ }_{, 3(N+2) 3} .
$$

But this required condition may be written

$$
{ }^{\prime} W^{A C}{ }_{3(N+4) 3}+\text { known terms }={ }^{\prime} S^{A C}{ }_{, 3(N+2) 3} .
$$

and here we have three equations which we can satisfy by simply reading off the values of

$$
{ }^{\prime} W^{A C}{ }_{3(N+4) 3} \text {. }
$$

From these values, and quantities already assigned, we obtain the appropriate values of the quantities (7.5) to satisfy (7.7).

We have thus carried the induction from $N$ to $(N+1)$, and so the Tensor Theorem is proved.

In the above treatment, which parallels that for the Vector Theorem, for brevity we omitted to treat (7.12) as a formal lemma, although the analogue was so treated in Sec. 4.

We are grateful to Dr. P. S. Florides for discussions.

REFERENCE

1. W. S. Dorn and A. Schild, Quart. Appl. Math. 14, 210, (1956) 